\title{
CYCLIC EXTENSIONS OF PARAFREE GROUPS ${ }^{1}$
}

BY

\author{
PENG-CHOON WONG
}

\begin{abstract}
Let $1 \rightarrow F \rightarrow G \rightarrow T \rightarrow 1$ be a short exact sequence where $F$ is parafree and $T$ is infinite cyclic. We examine some properties of $G$ when $F / F^{\prime}$ is a free $Z T$-module. Here $F^{\prime}$ is the commutator subgroup of $F$ and $Z T$ is the integral group ring of $T$. In particular, we show $G$ is parafree and $\gamma_{n} F / \gamma_{n+1} F$ is a free $Z T$-module for every $n>1$ (where $\gamma_{n} F$ is the $n$th term of the lower central series of $F$ ).
\end{abstract}

1. Introduction. A group $G$ is termed parafree if

(i) $G$ is residually nilpotent and

(ii) $G$ has the same lower central sequence as some free group.

This paper is mainly concerned with some of the module structure involved with the action of an infinite cyclic group on a parafree group. More precisely, let $1 \rightarrow F \rightarrow G \rightarrow T \rightarrow 1$ be a short exact sequence where $F$ is a parafree group and $T=\langle t\rangle$ is an infinite cyclic group generated by $t$. Here we assume $T$ to be a subgroup of $G$. If $F^{\prime}$ denotes the commutator subgroup of $F$ and $Z T$ denotes the integral group ring of $T$, then $F / F^{\prime}$ can be regarded as a $Z T$-module once the action of $t$ on $F / F^{\prime}$ is defined (by conjugation):

$$
a F^{\prime} * t=t^{-1} a t F^{\prime}, \quad a \in F .
$$

We shall prove

Theorem 1. Let $1 \rightarrow F \rightarrow G \rightarrow T \rightarrow 1$ be a short exact sequence where $F$ is parafree and $T=\langle t\rangle$ is infinite cyclic. If $F / F^{\prime}$ is a free $Z T$-module then $G$ is parafree

and

TheOREM 2. Let $1 \rightarrow F \rightarrow G \rightarrow T \rightarrow 1$ be a short exact sequence where $F$ is parafree and $T=\langle t\rangle$ is infinite cyclic. If $F / F^{\prime}$ is a free $Z T$-module then $\gamma_{n} F / \gamma_{n+1} F$ is a free $Z T$-module for every $n \geqslant 1$. (Here $\gamma_{n} F$ is the nth term of the lower central series of $F$.)

Such extensions $G$ discussed above are not rare. Indeed it is clear that if $G$ is free on $X \cup\{t\}$ and $F$ is the normal closure of $X$, then the short exact sequence $1 \rightarrow F \rightarrow G \rightarrow T \rightarrow 1$ where $T=\langle t\rangle$, satisfies the hypothesis of Theorems 1 and 2 .

Received by the editors May 10, 1978.

AMS (MOS) subject classifications (1970). Primary 20E05, 20E15; Secondary 20F25, 20 E25.

Key words and phrases. Free group, parafree groups, free nilpotent groups, free module, integral group ring.

'This paper is taken from the author's Ph.D. thesis, New York University, 1978, written under the guidance of Professor Gilbert Baumslag. 
Even in this case the result seems to be new and sheds additional light on the nature of free groups themselves.

Furthermore the one-relator groups $G=\left\langle a, b, t ; a=\left[t^{i}, a\right]\left[t^{j}, b\right]\right\rangle, i \cdot j \neq 0$, are also examples of such extensions (see §6).

Now according to G. Baumslag [7], the 2-generator subgroups of parafree groups are free. G. Baumslag's proof uses machinery from Lie algebras. For these parafree groups covered in Theorems 1 and 2 there is a straightforward proof which comes out of Theorem 2. Indeed we shall easily deduce the following corollary of Theorem 2.

THEOREM 3. Let $1 \rightarrow F \rightarrow G \rightarrow T \rightarrow 1$ be a short exact sequence where $F$ is parafree and $T=\langle t\rangle$ is infinite cyclic. If $F / F^{\prime}$ is a free $Z T$-module then every two-generator subgroup of $G$ is free.

Similarly we have

TheOREM 4. Let $1 \rightarrow F \rightarrow G \rightarrow T \rightarrow 1$ be a short exact sequence where $F$ is parafree and $T=\langle t\rangle$ is infinite cyclic. If $F / F^{\prime}$ is a finitely generated free $Z T$-module, freely generated by $\left\{c F^{\prime}, d F^{\prime}, \ldots, e F^{\prime}\right\}$, then the group $H=g p(t, c, d, \ldots, e)$ is free.

Many authors have discussed the existence, in a group satisfying appropriate conditions, of a finitely generated normal subgroup (see, for example, B. Baumslag [1], G. Baumslag [11], R. G. Burns [14], A. Karrass and D. Solitar [18]-[20]). In particular, O. Schreier [22] proved that if $F$ is a free group and $N$ is a nontrivial finitely generated normal subgroup of $F$, then $N$ is of finite index in $F$. The corresponding possibilities for parafree groups has not been investigated so far. Here we note, as an easy consequence of Theorem 2,

TheOREM 5. Let $1 \rightarrow F \rightarrow G \rightarrow T \rightarrow 1$ be a short exact sequence where $F$ is parafree and $T=\langle t\rangle$ is infinite cyclic. If $F / F^{\prime}$ is a free $Z T$-module and $N$ is a finitely generated normal subgroup of $G$ contained in $F$, i.e., $N \triangleleft G, N \leqslant F$, then $N$ is trivial, i.e., $N=1$.

The arrangement of the rest of this paper is as follows. In $\$ 2$ we prove Theorem 1. In $\$ 3$ we prepare the ground for the proof of Theorem 2 which takes up $\$ 4$. In $\$ 5$ we prove Theorems 3,4 and 5. Finally in $\$ 6$ we give an example of a nonfree parafree group satisfying the hypothesis of Theorem 1 .

The notations used here are common in the literature (see W. Magnus, A. Karrass and D. Solitar [21], G. Baumslag [8]). In particular, if $G$ is a group and $X$ is a subset of $G$, then $g p(X)$ and $g p_{G}(X)$ denote respectively the subgroup and the normal subgroup generated by $X$. Furthermore we have $[x, y]=x^{-1} y^{-1} x y, x^{y}=$ $y^{-1} x y$ for $x, y \in G$ and $Z$ denotes the ring of integers.

The author wishes to express his deepest appreciation to Professor Gilbert Baumslag for his invaluable help and guidance.

2. The proof of Theorem 1 . Theorem 1 follows immediately from the proof of the following theorem. 
THEOREM 1'. Let $1 \rightarrow F \rightarrow G \rightarrow T \rightarrow 1$ be a short exact sequence where $F$ is parafree and $T=\langle t\rangle$ is infinite cyclic. If $F / F^{\prime}$ is a finitely generated free $Z T$-module, then $G$ is parafree.

Proof. First we show that $G$ is residually a finite $p$-group for all primes $p$.

Let $p$ be a prime. We can assume $p \neq 2$ since the proof for $p=2$ is similar. Let $g \in G, g \neq 1$. We have to construct a homomorphism $\phi$ of $G$ onto a finite $p$-group such that $g \phi \neq 1$.

If $g \notin F$, we can easily construct the homomorphism.

Now suppose $g \in F$. We choose an integer $k \geqslant 1$ so that $g \notin \gamma_{k+1} F$. Observe that $F / \gamma_{k+1} F$ is a free nilpotent group of class $k$ and hence a residually finite $p$-group. Then there exist a normal subgroup $R$ of $F / \gamma_{k+1} F$ such that $g \gamma_{k+1} F \notin R$ and $\left(F / \gamma_{k+1} F\right) / R$ has order $p^{d}, d>1$. This implies $g \gamma_{k+1} F \notin\left(F / \gamma_{k+1} F\right)^{p^{d}}$ or equivalently, $g \notin\left(\gamma_{k+1} F\right) F^{p^{d}}$.

By hypothesis, $F / F^{\prime}$ is a finitely generated free $Z T$-module, freely generated say by

$$
c_{1,0} F^{\prime}, c_{2,0} F^{\prime}, \ldots, c_{n, 0} F^{\prime}
$$

Put

$$
c_{i, j}=c_{i, 0}^{\iota^{j}}, \quad 1 \leqslant i \leqslant n, j \in Z \text {. }
$$

Then modulo $\left(\gamma_{k+1} F\right) F^{p^{d}}$, the set $X=\left\{c_{i, j} \mid 1 \leqslant i \leqslant n, j \in Z\right\}$ freely generates $F /\left(\gamma_{k+1} F\right) F^{p^{d}}$ viewed as a free group in the variety of all nilpotent groups of class at most $k$ and exponent dividing $p^{d}$.

Now let us put
(a) $\bar{F}=F /\left(\gamma_{k+1} F\right) F^{p^{d}}$,
(b) $\bar{g}=g\left(\gamma_{k+1} F\right) F^{p^{d}}$,
(c) $\bar{c}_{i, j}=c_{i, j}\left(\gamma_{k+1} F\right) F^{p^{d}}, 1<i \leqslant n, j \in Z$,
(d) $\bar{X}=\left\{\bar{c}_{i, j} \mid 1 \leqslant i \leqslant n, j \in Z\right\}$.

Since $\bar{X}$ freely generates $\bar{F}$, there exists an integer $m$ such that the group

$$
\bar{N}=g p\left(\bar{c}_{i, j} \mid 1 \leqslant i \leqslant n,-\frac{\left(p^{m}-1\right)}{2} \leqslant j \leqslant \frac{\left(p^{m}-1\right)}{2}\right)
$$

contains $\bar{g}$. Since $\bar{N}$ is a finitely generated nilpotent group of class at most $k$ and exponent dividing $p^{d}, \bar{N}$ is a finite $p$-group.

We define an automorphism $\tau$ of $\bar{N}$ by

$$
\left.\begin{array}{lll}
\bar{c}_{i,-\left(p^{m}-1\right) / 2} \tau & = & \bar{c}_{i,-\left(p^{m}-1\right) / 2+1} \\
\bar{c}_{i,-\left(p^{m}-1\right) / 2+1} \tau & = & \bar{c}_{i,-\left(p^{m}-1\right) / 2+2} \\
\vdots & & \\
\bar{c}_{i, 0} \tau & = & \bar{c}_{i, 1} \\
\vdots & & \\
\bar{c}_{i,\left(p^{m}-1\right) / 2} \tau & = & \bar{c}_{i,-\left(p^{m}-1\right) / 2}
\end{array}\right\}, \quad 1 \leqslant i<n .
$$


It is obvious that $\tau$ is of order $p^{d}$. Now let $\bar{T}=\langle\bar{t}\rangle$ be a cyclic group of order $p^{d}$ generated by $\bar{t}$. Finally let $\bar{G}$ be the split extension of $\bar{N}$ by $\langle\bar{t}\rangle$ with $\bar{t}$ inducing the automorphism $\tau$ on $\bar{N}$, i.e.,

$$
\bar{G}=g p\left(\bar{N}, \bar{t} ; \bar{t}^{-1} u \bar{t}=u \tau, u \in \bar{N}\right) .
$$

The group $\bar{G}$ is clearly a finite $p$-group.

It is now clear that the mapping $\phi$ of $G$ onto $\bar{G}$ defined by

$$
\phi: t \rightarrow \bar{t}, \quad c_{i, 0} \rightarrow \bar{c}_{i, 0}, \quad 1 \leqslant i<n,
$$

extends to a homomorphism of $G$ onto $\bar{G}$ which we again denote by $\phi$. Notice that $g \phi=\bar{g} \neq 1$. Hence $G$ is residually a finite $p$-group.

Now, we show that $G$ has the same lower central sequence as a free group.

Let $X^{\prime}=\left\{c_{1,0}^{\prime}, c_{2,0}^{\prime}, \ldots, c_{n, 0}^{\prime}\right\}$ and $A$ be the free group freely generated by

$$
X^{\prime} \cup\left\{t^{\prime}\right\}=\left\{t^{\prime}, c_{1,0}^{\prime}, c_{2,0}^{\prime}, \ldots, c_{n, 0}^{\prime}\right\}
$$

We define a map $\theta$ from $A$ to $G$ by

$$
\phi: t^{\prime} \rightarrow t, \quad c_{i, 0}^{\prime} \rightarrow c_{i, 0}, \quad 1 \leqslant i \leqslant n .
$$

Then $\theta$ extends to a homomorphism, also denoted $\theta$, from $A$ to $G$. We claim $G$ has the same lower central sequence as $A$.

To see this, put $B=g p_{A}\left(X^{\prime}\right)$, i.e., $B$ is the normal closure of $X^{\prime}$ in $A$. Then $B$ is free on $Y=\left\{c_{i, j}^{\prime}=\left(t^{\prime}\right)^{-j} c_{i, 0}^{\prime}\left(t^{\prime}\right)^{j} ; \quad 1 \leqslant i \leqslant n, j \in Z\right\}$. Observe that $\theta$ induces a homomorphism of $B$ into $F$ and hence noting the actual effect of $\theta$ on the free set of generators $Y$ of $B$, it follows that $\theta$ induces an isomorphism of $B / \gamma_{2} B$ to $F / \gamma_{2} F$. Since $F$ is parafree, it follows that $\theta$ induces an isomorphism of $B / \gamma_{k+1} B$ to $F / \gamma_{k+1} F$ for every $k \geqslant 1$.

We next show $A / \gamma_{k+1} B$ is isomorphic to $G / \gamma_{k+1} F$. To do this let $\theta_{1}$ be homomorphism of $A$ to $G / \gamma_{k+1} F$ induced by $\theta$. We show $\operatorname{ker} \theta_{1}=\gamma_{k+1} B$. It is obvious that $\gamma_{k+1} B \leqslant \operatorname{ker} \theta_{1}$. Now let $x \in \operatorname{ker} \theta_{1}$. If $x \in B$, then $x \in \gamma_{k+1} B$ by the observation in the preceding paragraph. Suppose $x \in A, x \notin B$. Then $x$ can be written uniquely $x=t^{\prime m} w\left(\mathbf{c}_{i, j}^{\prime}\right)$ where $m \neq 0$ and $w\left(\mathbf{c}_{i, j}^{\prime}\right)$ is a word in the $c_{i, j}^{\prime}$ 's. Then

$$
x \theta_{1}=t^{m} w\left(c_{i, j}\right) \gamma_{k+1} F \neq \gamma_{k+1} F .
$$

Hence it follows that $\operatorname{ker} \theta_{1} \leqslant \gamma_{k+1} B$. Therefore $A / \gamma_{k+1} B \cong G / \gamma_{k+1} F$ and hence

$$
\begin{aligned}
A / \gamma_{k+1} A & \cong A / \gamma_{k+1} B / \gamma_{k+1}\left(A / \gamma_{k+1} B\right) \\
& \cong G / \gamma_{k+1} F / \gamma_{k+1}\left(G / \gamma_{k+1} F\right) \cong G / \gamma_{k+1} G .
\end{aligned}
$$

This completes the proof of the theorem.

3. Preparations for the proof of Theorem 2. We shall develop some notions from the commutator calculus (see G. Baumslag [8], P. Hall [16]). Unless stated differently, we assume the integer $n \geqslant 1$.

Let $F$ be a group, not necessarily free, and equipped with a fixed set of generators $X=\left\{x_{1}, x_{2}, \ldots, x_{q}\right\}, q<\infty$.

Definition 1. Commutation. We define a binary operation $\circ$, termed commutation, in $F$ by $g \circ h=[g, h], g, h \in F$. 
Definition 2. Commutators of weight $n, n \geqslant 1$. We define a commutator of weight $n, n \geqslant 1$, in $x_{1}, x_{2}, \ldots, x_{q}$ inductively as follows:

(i) $x_{1}, x_{2}, \ldots, x_{q}$ are the commutators of weight 1 ;

(ii) if $u, v$ are commutators of weight $i, j$ respectively and $i+j=n$, then $u \circ v$ is a commutator of weight $n$ in $x_{1}, x_{2}, \ldots, x_{q}$.

Remarks. (i) $(F, \circ)$ is a groupoid but not a semigroup since commutation is not associative in general;

(ii) The commutators of weight $n$ belong to $\gamma_{n} F$;

(iii) If $F$ is the free group on $x_{1}, x_{2}, \ldots, x_{q}$ then the weight of a commutator $c$ in $x_{1}, x_{2}, \ldots, x_{q}$ will, in general, differ from the length of $c$ viewed as an element in the free group $F$. For example the weight of $x_{1} \circ x_{2}$ is two but the length of $x_{1} \circ x_{2}$ is four.

Next we define free groupoid.

Definition 3. Free groupoid. Let $Y=\left\{y_{1}, y_{2}, \ldots, y_{q}\right\}, 1 \leqslant q<\infty$, be a set of symbols. We define a groupoid-word of length $n, n>1$, in $y_{1}, y_{2}, \ldots, y_{q}$ inductively as follows:

(i) $y_{1}, y_{2}, \ldots, y_{q}$ are the groupoid-words of length 1;

(ii) if $u, v$ are groupoid-words of length $i, j$ respectively and $i+j=n$, then $u \circ v=(u v)$ is a groupoid-word of length $n$ in $y_{1}, y_{2}, \ldots, y_{q}$ and (uv) is just the juxtaposition of $u$ and $v$ with brackets.

Let $(P, \circ)$ be the collection of all groupoid-words of length $n, n>1$. Then $(P, \circ)$ is defined to be the free groupoid on $y_{1}, y_{2}, \ldots, y_{q}$.

Now we define the form of a commutator by using Definition 3.

Definition 4. Form of a commutator. Let $(F, \circ)$ be defined as in Definition 1 above and let $(M, \circ)$ be the free groupoid on $x$. We define, inductively, a map $\phi$ from the set of commutators of weight $n, n>1$, in $x_{1}, x_{2}, \ldots, x_{q}$ into $\left(M,{ }^{\circ}\right)$ as follows:

(i) if $x_{i}, 1 \leqslant i \leqslant q$, is a commutator of weight 1 , then $x_{i} \phi=x, 1<i \leqslant q$;

(ii) if $w=u \circ v$ is a commutator of weight $n, n \geqslant 2$, where $u, v$ are commutators of weight $i, j$ respectively and $i+j=n$, then $w \phi=(u \circ v) \phi=u \phi \circ v \phi$.

We call the image $w \phi$ of the commutator $w$, the form of $w$.

REMARKs. Since $(M, \circ)$ is a free groupoid we have the following observations:

(i) the form of a commutator of weight $n$ is unique;

(ii) the form of a commutator $w$ of weight $n$, depends upon the order in which $w$ is formed;

(iii) if $u, v$ are any two commutators of a fixed weight $n$, we write $u \sim v$ if and only if $u$ and $v$ have the same form, i.e., $u \phi=v \phi$. Then $\sim$ is an equivalence relation. Hence the set of commutators of a fixed weight $n$ can be divided into disjoint subsets where each subset contains commutators of a fixed form.

Notation. We will adopt the following notations for the remainder of $\$ \S 3,4$. Let $F$ be a group.

(i) If $g_{1}, g_{2}, \ldots, g_{n} \in F, n<\infty$, we write $g_{1} \circ g_{2} \circ g_{3} \circ \ldots \circ g_{n}=$ $\left(\cdots \cdot\left(\left(g_{1} \circ g_{2}\right) \circ g_{3}\right) \circ \cdots \circ g_{n}\right)$.

(ii) If $g$ is a commutator of weight $n$, we denote the weight of $g$ by $|g|$; so here $|g|=n$. 
The above two notations are not standard but turn out to be convenient here.

Theorem 2 will depend in large measure on a carefully selected sequence of elements of $F$ (called a basic sequence). From this sequence the free generators of $\gamma_{n} F / \gamma_{n+1} F$ will emerge. More precisely, let $F$ be a free group freely generated by a finite set $X=\left\{x_{1}, x_{2}, \ldots, x_{q}\right\}, q<\infty$.

Definition 5. Basic sequence. A sequence $b_{1}, b_{2}, \ldots$ of commutators in $x_{1}$, $x_{2}, \ldots, x_{q}$ (viewed as elements in the groupoid $(F, \circ)$ ) is called a basic sequence if the following conditions are satisfied:

(i) every element of $X$ occurs in the sequence;

(ii) if $\left|b_{i}\right|<\left|b_{j}\right|$, then $i<j$;

(iii) if $b_{r}$ is of weight at least two, then

(a) $b_{r}=b_{i} \circ b_{m}, m<i$ and

(b) either $\left|b_{i}\right|=1$ or $b_{r}=b_{k} \circ b_{l} \circ b_{m}$ where $k>l, m \geqslant l$ and $b_{k}, b_{l}$ and $b_{m}$ are of smaller weights than $b_{r}$.

Definition 6. Basic commutator. The elements $b_{1}, b_{2}, \ldots$ in a basic sequence are called basic commutators and the index of the basic commutator $b_{j}$ is defined to be $j$.

We now construct a basic sequence in $F$.

LEMMA 1. Let $F$ be a free group freely generated by a finite set $X=$ $\left\{x_{1}, x_{2}, \ldots, x_{q}\right\}, q<\infty$. Then a basic sequence $b_{1}, b_{2}, \ldots$ in $F$ exists. Furthermore, the basic sequence can be arranged in the following way:

(3.1) if $b_{i}$ is the ith term in the basic sequence and $i \leqslant q$, then $b_{i}=x_{i}$, i.e., the indices of $b_{i}$ and $x_{i}$ are the same for $i=1,2, \ldots, q$.

(3.2) if $b_{i}=b_{l_{i}} \circ b_{m_{i}}$ and $b_{j}=b_{l_{j}} \circ b_{m_{j}}$ are both basic commutators of weight 2 , then $i<j$ if and only if one of the following holds:

(i) $l_{i}<l_{j}$

(ii) $l_{i}=l_{j}, m_{i}<m_{j}$.

(3.3) if $b_{i}=b_{k_{i}} \circ b_{l_{i}} \circ b_{m_{i}}$ and $b_{j}=b_{k_{j}} \circ b_{l_{j}} \circ b_{m_{j}}$ are both basic commutators of weight $n, n>2$, then $i<j$ if and only if one of the following holds:

(i) $\left|b_{m_{i}}\right|<\left|b_{m_{j}}\right|$;

(ii) $\left|b_{m_{i}}\right|=\left|b_{m_{j}}\right|,\left|b_{l_{i}}\right|<\left|b_{l_{j}}\right|$;

(iii) $\left|b_{m_{i}}\right|=\left|b_{m_{j}}\right|,\left|b_{l_{i}}\right|=\left|b_{l_{j}}\right|, k_{i}<k_{j}$;

(iv) $\left|b_{m_{i}}\right|=\left|b_{m_{j}}\right|,\left|b_{l_{i}}\right|=\left|b_{l_{j}}\right|, k_{i}=k_{j}, l_{i}<l_{j}$;

(v) $\left|b_{m_{i}}\right|=\left|b_{m_{j}}\right|,\left|b_{l_{i}}\right|=\left|b_{l_{j}}\right|, k_{i}=k_{j}, l_{i}=l_{j}, m_{i}<m_{j}$.

Proof. The following method will be used in constucting the basic sequence in $F$ (see G. Baumslag [8, p. 30]).

Let $A$ be a subset of $F$ and let $b \in A$. Define

$$
A \operatorname{rep} b=\{a i b \mid i=0,1,2, \ldots, a \in A \backslash b\}
$$

where

$$
a \circ b=a \text { and } a(i+1) b=(a i b) \circ b .
$$

Thus $A$ rep $b$ consists of all elements of $A$ excluding $b$ and those obtained from them by repeated commutation on the right by $b$. 
We now define a basic sequence $b_{1}, b_{2}, \ldots$ in $F$. Put $b_{1}=x_{1}, b_{2}=x_{2}, \ldots, b_{q}$ $=x_{q}$ and $X_{1}=X, X_{2}=X_{1} \operatorname{rep} b_{1}, \ldots, X_{q}=X_{q-1}$ rep $b_{q-1}$. Suppose inductively, $b_{q+1}, \ldots, b_{q+r}$ have been defined and that

$$
X_{q+i}=X_{q+i-1} \operatorname{rep} b_{q+i-1} \quad(i=1,2, \ldots, r+1)
$$

so that conditions (3.2) and (3.3) are satisfied.

We have to define $b_{q+r+1}$. Observe that since $q<\infty$, each $X_{i}, i>1$, contains only finitely many commutators of any fixed weight. Consider the finitely many commutators of the smallest weight in $X_{q+r+1}$. We now carefully select one of them to be $b_{q+r+1}$.

We note first that, using only the information above, it is now easy to verify that $b_{1}, b_{2}, \ldots$ is a basic sequence in $F$, i.e., the conditions of Definition 4 are satisfied.

Continuing the proof, let $\mathcal{C}$ be any set of commutators. Define

$$
\mu(\mathcal{C})=\operatorname{minimum}(|c| \mid c \in \mathcal{C})
$$

where $|c|$ is the weight of $c$.

We note, now that there are two possibilities in selecting $b_{q+r+1}$.

(I) $\mu\left(X_{q+r+1}\right)=\mu\left(X_{q+r}\right)$. In this case, the commutators of smallest weight in $X_{q+r+1}$ are also the commutators of smallest weight in $X_{q+r}$. Hence, by induction, they have been ordered to satisfy one of the conditions (3.2), (3.3). Define $b_{q+r+1}$ to be the first of these commutators in $X_{q+r+1}$.

(II) $\mu\left(X_{q+r+1}\right)>\mu\left(X_{q+r}\right)$. There are two subcases.

(a) $\mu\left(X_{q+r+1}\right)=2$. Then the commutators of smallest weight in $X_{q+r+1}$ are of the form $b_{l} \circ b_{m}, l>m$ and $\left|b_{l}\right|=\left|b_{m}\right|=1$. We order these commutators $b_{l} \circ b_{m}$ according to (3.2) and denote the ordering by $\alpha$. More precisely, if $b_{l_{i}} \circ b_{m_{i}}$ and $b_{l j} \circ b_{m_{j}}$ are any two commutators of smallest weight in $X_{q+r+1}$, then $b_{l_{i}} \circ b_{m_{i}} \alpha$ $b_{l j} \circ b_{m j}$ if and only if one of the following holds:

(i) $l_{i}<l_{j}$;

(ii) $l_{i}=l_{j}, m_{i}<m_{j}$.

Now choose $b_{q+r+1}$ to be the first of these commutators of smallest weight in $X_{q+r+1}$.

(b) $\mu\left(X_{q+r+1}\right)>2$. Then the commutators of smallest weight in $X_{q+r+1}$ are of the form $b_{k} \circ b_{l} \circ b_{m}$ where $k>l, m \geqslant l$ and $b_{k}, b_{l}$ and $b_{m}$ are of smaller weights than $\mu\left(X_{q+r+1}\right)$. By induction, the $b_{k}$ 's, $b_{l}$ 's and $b_{m}$ 's are well ordered and hence their indices are well defined.

We define an ordering, denoted $\alpha$, on these commutators $b_{k} \circ b_{l} \circ b_{m}$ of smallest weight according to (3.3). More precisely, if $b_{k_{i}} \circ b_{l_{i}} \circ b_{m_{i}}$ and $b_{k_{j}} \circ b_{l_{j}} \circ b_{m_{j}}$ are any two commutators of smallest weight in $X_{q+r+1}$, then $b_{k_{i}} \circ b_{l_{i}} \circ b_{m_{i}} \alpha b_{k_{j}} \circ b_{l_{j}} \circ b_{m_{j}}$ if and only if one of the following holds:

(i) $\left|b_{m_{1}}\right|<\left|b_{m_{j}}\right|$;

(ii) $\left|b_{m_{i}}\right|=\left|b_{m_{j}}\right|,\left|b_{l_{i}}\right|<\left|b_{l_{j}}\right|$;

(iii) $\left|b_{m_{i}}\right|=\left|b_{m_{j}}\right|,\left|b_{l_{i}}\right|=\left|b_{l_{j}}\right|, k_{i}<k_{j}$;

(iv) $\left|b_{m_{i}}\right|=\left|b_{m_{j}}\right|,\left|b_{l_{i}}\right|=\left|b_{l_{j}}\right|, k_{i}=k_{j}, l_{i}<l_{j}$;

(v) $\left|b_{m_{i}}\right|=\left|b_{m_{j}}\right|,\left|b_{l_{i}}\right|=\left|b_{l_{j}}\right|, k_{i}=k_{j}, l_{i}=l_{j}, m_{i}<m_{j}$.

Now choose $b_{q+r+1}$ to be the first of these commutators in $X_{q+r+1}$. 
It is easy to see now that the basic sequence $b_{1}, b_{2}, \ldots$ satisfies (3.1), (3.2) and (3.3). This completes the proof of the lemma.

COROLlaRY 1. The basic commutators of the same weight and same form (as constructed in Lemma 1) are arranged lexicographically.

LEMMA 2. Let $F$ be a free group of finite rank. Let $b_{1}, b_{2}, \ldots$ be a basic sequence of $F$ and $B_{n}$ be the set of basic commutators of weight $n$, i.e., $B_{n}=\left\{b_{j}|| b_{j} \mid=n\right\}$. Then $\gamma_{n} F / \gamma_{n+1} F$ is a free abelian group freely generated by the elements of $B_{n}$.

Proof. For a proof refer to G. Baumslag [8], P. Hall [16].

We have so far constructed a particular basic sequence for a free group of finite rank. We now extend the results to a free group $F$ of infinite rank equipped with a free set $X$ of generators where $X=\left\{c_{i}, d_{i}, e_{i}, \ldots ;(i \in Z)\right\}$. For ease of exposition we shall restrict our attention to the case where $X=\left\{c_{i}, d_{i} ;(i \in Z)\right\}$. The case where there are further "batches" of generators $e_{i}, \ldots(i \in Z)$ can be handled in much the same way.

LEMMA 3. Let $F=\left\langle c_{i}, d_{i} ; i \in Z\right\rangle$ be a free group freely generated by $c_{i}, d_{i}, i \in Z$. Let $F_{j}, j \geqslant 0$, be the subgroup of $F$ generated by

$$
c_{-j}, \ldots, c_{0}, \ldots, c_{j}, d_{-j}, \ldots, d_{0}, \ldots, d_{j}
$$

and $B_{j, n}$ be the set of basic commutators of weight $n$ in $F_{j}$. Put $B_{n}=\cup_{j=0}^{\infty} B_{j, n}$. Then modulo $\gamma_{n+1} F, \gamma_{n} F$ is a free abelian group freely generated by the elements of $B_{n}$.

Proof. Notice first that, modulo $\gamma_{n+1} F_{j}, \gamma_{n} F_{j}, j \geqslant 0$, is a free abelian group freely generated by the elements of $B_{j, n}$ (Lemma 2).

We show $B_{n}$ spans $\gamma_{n} F$ modulo $\gamma_{n+1} F$. To this end, let $x \in \gamma_{n} F$. Then $x \in F_{j}$ for some $j>0$. Now $F_{j}$ is a free factor of $F$ which implies that

$$
\gamma_{r} F \cap F_{j}=\gamma_{r} F_{j} \quad(r=1,2, \ldots) .
$$

So $x \in \gamma_{n} F_{j}$ which means that $x \in g p\left(B_{j, n}\right)$ (modulo $\left.\gamma_{n+1} F_{j}\right)$. Therefore $x \in g p\left(B_{n}\right)$ (modulo $\gamma_{n+1} F$ ).

In order to prove the elements of $B_{n}$ are independent modulo $\gamma_{n+1} F$, it suffices to prove that the elements of $B_{j, n}(j=0,1,2, \ldots)$ are independent modulo $\gamma_{n+1} F$. But this follows immediately from equation (3.4) and the fact that the elements of $B_{j, n}$ are independent modulo $\gamma_{n+1} F_{j}$.

LEMMA 4. Let $F=\left\langle c_{i}, d_{i} ; i \in Z\right\rangle$ be a free group freely generated by $c_{i}, d_{i}, i \in Z$. Let $F_{j}$ and $B_{j, n}$ be as defined in Lemma 3. Put $B=\cup_{j, n} B_{j, n}$. Then the elements of $B$ can be totally ordered by using Lemma 1.

Proof. For each $j=0,1,2, \ldots$ we order (denoted $<_{j}$ ) the generators of $F_{j}$ as follows:

$$
c_{-j}<_{j} c_{-j+1}<_{j} \cdots<_{j} c_{0}<_{j} \cdots<_{j} c_{j}<_{j} d_{-j}<_{j} \cdots<_{j} d_{0}<_{j} \cdots<_{j} d_{j}
$$

Now put $B_{j, \infty}=\bigcup{ }_{n=1}^{\infty} B_{j, n}$. The orderings extend by Lemma 1 to a total order (denoted $<_{j}$ ) of $B_{j, \infty}$. So each $B_{j, \infty}$ becomes a totally ordered set. Notice that $<_{j+1} \mid B_{j, \infty}=<_{j}$ and so we can define an ordering $<$ on $B$ by $<=\cup_{j=0}^{\infty}<_{j}$, i.e., if 
$x, y \in B$, then $x, y \in B_{j, \infty}$ for some $j \geqslant 0$ and by definition $x \leqslant y$ if and only if $x \leqslant j y$.

It follows that the order relation $\leqslant$ on $B$ is a total order. This completes the proof of the lemma.

We call the elements of $B_{n}$ the basic commutators of weight $n$ of $F$.

We next extend our definition of form (Definition 3 ) of a basic commutator to the case when $F$ is a free group freely generated by $c_{i}, d_{i}, i \in Z$.

Let $F_{j}$ be the subgroup of $F$ generated by $c_{-j}, \ldots, c_{0}, \ldots, c_{j}$, $d_{-j}, \ldots, d_{0}, \ldots, d_{j}$ and $\left(F_{j}\right.$, $\left.{ }^{\circ}\right)$ as before (Definition 2 and its remarks). Let $(N, \circ)$ be the free groupoid on $c, d$. We define, inductively, a map $\theta_{j}$ from the set of commutators of weight $n, n \geqslant 1$, in $c_{i}, d_{i},-j \leqslant i \leqslant j$, of $F_{j}$ into $\left(N,{ }^{\circ}\right)$ as follows:

(i) if $c_{i}, d_{i},-j \leqslant i \leqslant j$, is a commutator of weight 1 , then

$$
c_{i} \theta_{j}=c, \quad-j \leqslant i \leqslant j ; \quad d_{i} \theta_{j}=d, \quad-j \leqslant i \leqslant j ;
$$

(ii) if $w=u \circ v$ is a commutator of weight $n, n \geqslant 2$, where $u, v$ are commutators of weight $p, q$ respectively and $p+q=n$, then $w \theta_{j}=u \theta_{j} \circ v \theta_{j}$. Notice that $\theta_{j+1} \mid F_{j}$ $=\theta_{j}$.

DEFinition 7. Macro-form of a commutator. Let $F$ be the free group freely generated by $c_{i}, d_{i}, i \in Z$, and $F_{j}$ the subgroup of $F$ generated by

$$
c_{-j}, \ldots, c_{0}, \ldots, c_{j}, d_{-j}, \ldots, d_{0}, \ldots, d_{j} \text {. }
$$

Let $(N, \circ)$ be the free groupoid on $c, d$. We define a map $\theta$ from $(F, \circ)$ to $(N, \circ)$ by $\theta=\cup{ }_{j=0}^{\infty} \theta_{j}$ where $\theta_{j}$ is as above, i.e., if $x \in F$, then $x \in F_{j}$ for some $j>0$ and by definition $x \theta=x \theta_{j}$. Then $x \theta$ is unique and we call $x \theta$ the macro-form of $x$.

CoRollary 2. For $F=\left\langle c_{i}, d_{i} ; i \in Z\right\rangle$ be a free group freely generated by $c_{i}, d_{i}$, $i \in Z$. Then the basic commutators of the same weight and same macro-form (as constructed in Lemma 3) are ordered lexicographically.

REMARKS. (i) It is convenient to relabel the element $r \in B$ (see Lemma 4) by $b_{r}$ and call $r$ the index of $b_{r}$. So, of course, we have

$$
b_{r} \leqslant b_{s} \text { if and only if } r<s, r, s \in B \text {. }
$$

(ii) From Lemma 1 we have

(a) if $b_{k} \circ b_{l}$ is a basic commutator of weight 2 , then $b_{k}>b_{l}$,

(b) if $b_{k} \circ b_{l} \circ b_{m}$ is a basic commutator of weight $n, n>2$, then $b_{k}>b_{l}$ and $b_{m}>b_{l}$.

4. The proof of Theorem 2. Theorem 2 follows immediately from the slightly stronger

TheOrem 2'. Let $1 \rightarrow F \rightarrow G \rightarrow T \rightarrow 1$ be a short exact sequence where $F$ is free nilpotent and $T=\langle t\rangle$ is infinite cyclic. If $F / F^{\prime}$ is a finitely generated free $Z T$-module then $\gamma_{n} F / \gamma_{n+1} F$ is also a free ZT-module for every $n>1$. 
Proof. The proof is by induction on the class $r$ of $F$. The hypothesis is the case $r=1$. So we can assume $r>1$ and the result holds for $\gamma_{k} F / \gamma_{k+1} F, 1<k<r-1$.

By hypothesis $F / F^{\prime}$ is a finitely generated free $Z T$-module of rank $s$, freely generated by say $c F^{\prime}, d F^{\prime}, \ldots, e F^{\prime}$. For ease of exposition we restrict our attention to the case where $s=2$, i.e., $F / F^{\prime}$ is freely generated as a $Z T$-module by $c F^{\prime}, d F^{\prime}$.

Now let $c_{i}=t^{-i} c t^{i}$ and $d_{i}=t^{-i} d t^{i}, i \in Z$. Then modulo $F^{\prime}, c_{i}, d_{i}, i \in Z$, generate $F$ and $c_{i}, d_{i}, i \in Z$, are linearly independent. Since $F$ is free nilpotent of class $r$, this implies $c_{i}, d_{i}, i \in Z$, freely generate $F$. Then by Lemma $3, \gamma_{r} F$ is a free abelian group freely generated by the basic commutators of weight $r$.

We shall show $\gamma_{r} F$ is a free $Z T$-module by exhibiting a free set of generators for it.

We introduce the notation $*$ for the scalar multiplication in $\gamma_{k} F / \gamma_{k+1} F$ (qua $Z T$-module), $1 \leqslant k \leqslant r$, where

$$
x \gamma_{k+1} F * t^{i}=t^{-i} x t^{i} \gamma_{k+1} F, \quad x \in \gamma_{k} F, i \in Z \text {. }
$$

Furthermore we shall adopt the notations of $\$ 3$.

The case when the class $r=2$. In this case, the basic commutators of weight 2 in $\gamma_{2} F$ are of the form $b_{k} \circ b_{l}, k>l, k, l \in B$ and $\left|b_{k}\right|=\left|b_{l}\right|=1$. We denote the set of basic commutators of weight 2 by $B_{2}$.

Furthermore, notice that the basic commutators of weight 1 are simply the elements $c_{i}$ and $d_{i}, i \in Z$. It follows from the ordering of the basic commutators of weight 1 , i.e.,

$$
\cdots<c_{-1}<c_{0}<c_{1}<\cdots<\cdots<d_{-1}<d_{0}<d_{1}<\cdots
$$

that the elements of $B_{2}$ can be divided, according to their macro-forms into the following disjoint subsets:

$$
\begin{gathered}
R_{1}=\left\{c_{i} \circ c_{j} \mid i>j, i, j \in Z\right\}, \quad R_{2}=\left\{d_{u} \circ c_{v} \mid u, v \in Z\right\} \\
R_{3}=\left\{d_{i} \circ d_{j} \mid i>j, i, j \in Z\right\}
\end{gathered}
$$

i.e., $B_{2}=R_{1} \cup R_{2} \cup R_{3}$.

Now observe that modulo $\gamma_{2} F$, the elements $c_{0}$ and $d_{0}$ from a free set of generators for the $Z T$-module $F / F^{\prime}$. Furthermore notice that

$$
c_{0} F^{\prime} * t^{p}=c_{p} F^{\prime}, \quad p \in Z ; \quad d_{0} F^{\prime} * t^{p}=d_{p} F^{\prime}, \quad p \in Z
$$

We shall make use of the above trivial observation to prove that $\gamma_{2} F$ is a free $Z T$-module. So let

$$
T_{1}=\left\{c_{m} \circ c_{0} \mid m>0\right\}, \quad T_{2}=\left\{d_{m} \circ c_{0} \mid m \in Z\right\}, \quad T_{3}=\left\{d_{m} \circ d_{0} \mid m>0\right\},
$$

and put $S_{2}=T_{1} \cup T_{2} \cup T_{3}$. We claim that $S_{2}$ is a free set of generators for the $Z T$-module $\gamma_{2} F$.

Lemma 5. $S_{2}=T_{1} \cup T_{2} \cup T_{3}$ generates the $Z T$-module $\gamma_{2} F$.

Proof. Observe that

$$
\begin{array}{ll}
c_{m} \circ c_{0} * t^{p}=c_{m+p} \circ c_{p}, & m>0, p \in Z, \\
d_{m} \circ c_{0} * t^{p}=d_{m+p} \circ c_{p}, & m, p \in Z, \\
d_{m} \circ d_{0} * t^{p}=d_{m+p} \circ d_{p}, & m>0, p \in Z .
\end{array}
$$


The right-hand sides of the equations (4.1) comprise the whole of $B_{2}$. Hence the lemma.

LEMMA 6. $T_{i}$ is linearly independent over $Z T$ for $i=1,2,3$.

Proof. We shall discuss only the case $i=1$. The other cases are similar and will be left to the reader. Suppose then

$$
c_{m_{1}} \circ c_{0} * f_{m_{1}}+c_{m_{2}} \circ c_{0} * f_{m_{2}}+\cdots+c_{m_{n}} \circ c_{0} * f_{m_{n}}=0
$$

where $0<m_{1}<m_{2}<\cdots<m_{n}$ and $f_{m_{i}} \in Z T, i=1,2, \ldots, n$. We may suppose $f_{m_{1}} \neq 0$. On multiplying equation (4.2) by a suitable power of $t$, we may assume that $f_{m_{1}}$ has the form

$$
f_{m_{1}}=a_{0}+a_{1} t+\cdots+a_{j} t^{j}, \quad j \geqslant 0, a_{0} \neq 0 .
$$

Then, by virtue of equation (4.1), the basic commutator $c_{m_{1}} \circ c_{0}$ has coefficient $a_{0}$ $(\neq 0)$ and arises once and only once in equation (4.2). Hence from the $Z$-linear independence of the elements of $B_{2}$, we must have $f_{m_{1}}=0$. This contradicts the choice of $f_{m_{1}}$ at the outset and so the lemma follows.

LEMMA 7. $S_{2}=T_{1} \cup T_{2} \cup T_{3}$ is linearly independent over $Z T$.

Proof. Suppose that

$$
\sum_{m} c_{m} \circ c_{0} * f_{m}+\sum_{u} d_{u} \circ c_{0} * f_{u}+\sum_{v} d_{v} \circ d_{0} * f_{v}=0
$$

where the $i$ th summation sign represents a finite $Z T$-linear sum of elements of $T_{i}$, $i=1,2,3$.

Then, by virtue of equation (4.1) and the $Z$-linear independence of the elements of $B_{2}$, it follows that

$$
\sum_{m} c_{m} \circ c_{0} * f_{m}=\sum_{u} d_{u} \circ c_{0} * f_{u}=\sum_{v} d_{v} \circ d_{0} * f_{v}=0
$$

Now by Lemma 6, we have $f_{m}=f_{u}=f_{v}=0$. Hence the lemma.

Thus we have proved that $S_{2}$ is a free set of generators for the $Z T$-module $\gamma_{2} F$.

The case when the class $r>2$. In this case the basic commutators of weight $r$ in $\gamma_{r} F$ are of the form $b_{k} \circ b_{l} \circ b_{m}, k>l, m>l, k, l, m \in B$ and $b_{k}, b_{l}, b_{m}$ are of weights less than $r$. We denote the set of basic commutators of weight $r$ by $B_{r}$.

Now, the elements of $B_{r}$ can be divided into disjoint subsets according to their macro-forms as follows (see the remarks following Definition 3):

$$
B_{r}=\bigcup_{\text {disjoint }} T_{\phi \psi}
$$

where

$$
\begin{array}{r}
T_{\phi \psi}=\left\{b_{k} \circ b_{l} \circ b_{m} \mid b_{k} \circ b_{l} \circ b_{m} \in B_{r}, b_{k} \circ b_{l} \text { has macro-form } \phi,\right. \\
\left.b_{m} \text { has macro-form } \psi\right\} .
\end{array}
$$

Notice that the macro-form of the element $b_{k} \circ b_{l} \circ b_{m}$ in $T_{\phi \psi}$ is $\phi \psi$. Notice further that the macro-forms $\phi \psi, \phi^{\prime} \psi^{\prime}$ are distinct unless $\phi, \phi^{\prime}$ are identical macro-forms and $\psi, \psi^{\prime}$ are identical macro-forms. 
We now construct, inductively, a free set of generators for the $Z T$-module $\gamma_{r} F$. To do so put

$$
S_{\phi \psi}=\left\{\begin{array}{l}
\bar{b}_{k} \circ \bar{b}_{l} \circ b_{m} \mid \bar{b}_{k} \circ \bar{b}_{l} \circ b_{m} \in T_{\phi \psi} \text { and } \bar{b}_{k} \circ \bar{b}_{l} \gamma_{i+1} F \text { ranges } \\
\text { over the already selected free set of generators } \\
\text { of macro-form } \phi \text { of the } Z T \text {-module } \gamma_{i} F / \gamma_{i+1} F, 1<i<r-1
\end{array}\right\} .
$$

Notice the definition of $S_{\phi \psi}$ makes tacit use of the obvious inductive assumptions. Now let

$$
S_{r}=\bigcup_{\text {disjoint }} S_{\phi \psi}
$$

Then we claim that $S_{r}$ is a free set of generators for the $Z T$-module $\gamma_{r} F$.

LEMMA 8. $T_{\phi \psi}=\bigcup_{n \in Z} S_{\phi \psi} * t^{n}$.

Proof. Let $b_{k} \circ b_{l} \circ b_{m} \in T_{\phi \psi}$ where $b_{k} \circ b_{l}$ and $b_{m}$ are basic commutators of weight $i$ and $j$ respectively with $1 \leqslant i, j<r$ and $i+j=r$. Hence, inductively in the $Z T$-module $\gamma_{i} F / \gamma_{i+1} F$, there exists an element $\bar{b}_{k_{1}} \circ \bar{b}_{l_{1}} \gamma_{i+1} F$ of the free set of generators such that

$$
b_{k} \circ b_{l} \gamma_{i+1} F=\bar{b}_{k_{1}} \circ \bar{b}_{l_{1}} \gamma_{i+1} F * t^{p}
$$

for some $p \in Z$. Now in the $Z T$-module $\gamma_{j} F / \gamma_{j+1} F$ observe that

$$
b_{m} \gamma_{j+1} F * t^{-p}=b_{m_{1}} \gamma_{j+1} F
$$

where $b_{m_{1}}$ is the appropriate basic commutator obtained by adding $-p$ to each index in $b_{m}$ (see also the comments following (i.b) below). Now form the commutator $\bar{b}_{k_{1}} \circ \bar{b}_{l_{1}} \circ b_{m_{1}}$ and note that

$$
b_{k} \circ b_{l} \circ b_{m}=\bar{b}_{k_{1}} \circ \bar{b}_{l_{1}} \circ b_{m_{1}} * t^{p} .
$$

It remains only to check $\bar{b}_{k_{1}} \circ \bar{b}_{l_{1}} \circ b_{m_{1}} \in S_{\phi \psi}$.

To do this we have to show

(i) $\bar{b}_{k_{1}} \circ \bar{b}_{l} \circ b_{m_{1}} \in T_{\phi \psi}$ and

(ii) $b_{k_{1}} \circ b_{l_{1}} \gamma_{i+1} F$ belongs to the free set of generators of the $Z T$-module $\gamma_{i} F / \gamma_{i+1} F$ where $1 \leqslant i \leqslant r-1$.

We need only to verify (i) since (ii) is already satisfied. To do so we prove

(i.a) $\bar{b}_{k_{1}} \circ \bar{b}_{l_{1}}$ has macro-form $\phi$ and $b_{m_{1}}$ has macro-form $\psi$,

(i.b) $\bar{b}_{k_{1}} \circ \bar{b}_{l_{1}} \circ b_{m_{1}}$ is a basic commutator of weight $r$, i.e., $k_{1}>l_{1}$ and $m_{1}>l_{1}$.

Now if $b$ is a commutator of weight $n$ and macro-form $\alpha$, then $b * t^{p}$ is also a commutator of weight $n$ and macro-form $\alpha$ for all $p \in Z$. So it follows that $\bar{b}_{k_{1}} \circ \bar{b}_{l_{1}}$ and $b_{k} \circ b_{l}$ have the same macro-form $\phi$ and $b_{m_{1}}$ and $b_{m}$ have the same macro-form $\psi$. Hence $\bar{b}_{k_{1}} \circ \bar{b}_{l_{1}} \circ b_{m_{1}}$ has macro-form $\phi \psi$. Furthermore, the weight of $\bar{b}_{k_{1}} \circ \bar{b}_{l_{1}} \circ b_{m_{1}}$ is obviously $r$.

Now if $b_{r}$ and $b_{s}$ are two basic commutators with $b_{r}<b_{s}$, then $b_{r} * t^{p}<b_{s} * t^{p}$ for all $p \in Z$ (see Corollary 2). Since $b_{k} \circ b_{l} \circ b_{m}$ is a basic commutator we have $b_{k}>b_{l}$ and $b_{m}>b_{l}$. Since $\bar{b}_{k_{1}}=b_{k} * t^{-p}, \bar{b}_{l_{1}}=b_{l} * t^{-p}$ and $b_{m_{1}}=b_{m} * t^{-p}$, it follows that $\bar{b}_{k_{1}}>\bar{b}_{l_{1}}$ and $b_{m_{1}}>\bar{b}_{l_{1}}$. In other words $k_{1}>l_{1}$ and $m_{1}>l_{1}$. 
Hence $\bar{b}_{k_{1}} \circ \bar{b}_{l_{1}} \circ b_{m_{1}} \in T_{\phi \psi}$ and the proof of the lemma is complete.

LEMMA 9. $S_{r}=\cup$ disjoint $S_{\phi \psi}$ generates the ZT-module $\gamma_{r} F$.

Proof. Since

$$
B_{r}=\bigcup_{\text {disjoint }} T_{\phi \psi}
$$

and $T_{\phi \psi}=\cup_{n \in Z} S_{\phi \psi} * t^{n}$ by Lemma 8, the result follows.

We observe that $S_{\phi \psi}$ can be further divided into disjoint subsets $S_{k}$, where

$$
S_{k \circ l}=\left\{\bar{b}_{k} \circ \bar{b}_{l} \circ b_{m} \mid \bar{b}_{k} \circ \bar{b}_{l} \circ b_{m} \in S_{\phi \psi} \text { and all the } \bar{b}_{k} \circ \bar{b}_{l} \text { are identical }\right\} \text {. }
$$

Hence

$$
S_{\phi \psi}=\bigcup_{\text {disjoint }} S_{k \circ l}
$$

Thus a finite $Z T$-linear sum of elements of $S_{\phi \psi}$ will be a $Z T$-linear sum of elements from a finite number of subsets $S_{k} \bullet$

LEMMA 10. $S_{\phi \psi}=U_{\text {disjoint }} S_{k_{\circ} l}$ is linearly independent over $Z T$.

Proof. Suppose that

$$
\begin{aligned}
\sum_{u} \bar{b}_{k_{1}} \circ \bar{b}_{l_{1}} \circ b_{u} * f_{u}+\sum_{v} & \bar{b}_{k_{2}} \circ \bar{b}_{l_{2}} \circ b_{v} * f_{v} \\
& +\cdots+\sum_{w} \bar{b}_{k_{s}} \circ \bar{b}_{l_{s}} \circ b_{w} * f_{w}=0
\end{aligned}
$$

is a finite $Z T$-linear sum of elements of $S_{\phi \psi}$ where the $i$ th summation sign represents a finite linear $Z T$-linear sum of elements of $S_{k_{1} \circ l_{i}}, i=1,2, \ldots, s$. Writing in full the first summation sign of equation (4.4) we get

$$
\begin{gathered}
\bar{b}_{k_{1}} \circ \bar{b}_{l_{1}} \circ b_{u_{1}} * f_{u_{1}}+\bar{b}_{k_{1}} \circ \overline{b_{l_{1}}} \circ b_{u_{2}} * f_{u_{2}}+\cdots+\bar{b}_{k_{1}} \circ \overline{b_{l_{1}}} \circ b_{u_{2}} * f_{u_{2}} \\
+\sum_{v} \overline{k_{k_{2}}} \circ \overline{b_{l_{2}}} \circ b_{v} * f_{v}+\cdots+\sum \overline{b_{k_{s}}} \circ \overline{b_{l}} \circ b_{w} * f_{w}=0
\end{gathered}
$$

where

(i) $u_{i} \in B, 1<i<z$,

(ii) $u_{1}<u_{2}<\cdots<u_{z}$,

(iii) $f_{u_{i}} \in Z T, 1<i<z$.

We may suppose $f_{u_{1}} \neq 0$. On multiplying equation (4.5) by a suitable power of $t$ we may assume that $f_{u_{1}}$ has the form

$$
f_{u_{1}}=a_{0}+a_{1} t+\cdots+a_{j} t^{j}, \quad j>0, a_{0} \neq 0 .
$$

Now $\bar{b}_{k_{i}} \circ \bar{b}_{l_{i}} * t^{p} \neq \bar{b}_{k_{1}} \circ \bar{b}_{l_{1}}$ for all $p \in Z, 2<i<s$, since $\left\{\bar{b}_{k_{1}} \circ \bar{b}_{l_{1}} \gamma_{i+1} F\right.$, $\left.\bar{b}_{k_{2}} \circ \bar{b}_{l_{2}} \gamma_{i+1} F, \ldots, \bar{b}_{k_{s}} \circ \bar{b}_{l_{s}} \gamma_{i+1} F\right\}$ is inductively part of a free set of generators of the appropriate $Z T$-module. It follows that the basic commutator $\bar{b}_{k_{1}} \circ \bar{b}_{l_{1}} \circ b_{u_{1}}$ has coefficient $a_{0}(\neq 0)$ and arises once and only once in equation (4.5). Hence from the $Z$-linear independence of the elements of $B_{r}$, we have $f_{u_{1}}=0$. This contradicts the choice of $f_{u_{1}}$ at the outset and so the lemma follows. 
LEMMA 11. $S_{r}=\cup_{\text {disjoint }} S_{\phi \psi}$ is linearly independent over $Z T$.

Proof. Suppose that

$$
\begin{aligned}
\sum \bar{b}_{k} \circ \bar{b}_{l} \circ b_{m} * f_{\alpha}+\sum \bar{b}_{u} \circ \bar{b}_{v} \circ b_{w} * f_{\beta} \\
\\
+\cdots+\sum \bar{b}_{x} \circ \bar{b}_{y} \circ b_{z} * f_{\gamma}=0
\end{aligned}
$$

is a finite $Z T$-linear sum of elements of $S_{r}$ where the first summation sign represents a finite $Z T$-linear sum of elements of $S_{\phi \psi}$, the second summation sign represents a finite $Z T$-linear sum of elements of $S_{\phi^{\prime} \psi^{\prime}}$, etc., and the macro-forms $\left\{\phi \psi, \phi^{\prime} \psi^{\prime}, \ldots\right\}$ are distinct.

Now notice that if $b_{k} \circ b_{l} \circ b_{m}$ has macro-form $\phi \psi$, then $b_{k} \circ b_{l} \circ b_{m} * t^{p}$ has macro-form $\phi \psi$ for all $p \in Z$ (see Lemma 8) and the elements of $B_{r}$ are linearly independent over $Z$. So it follows that

$$
\begin{aligned}
\sum \bar{b}_{k} \circ \bar{b}_{l} \circ b_{m} * f_{\alpha} & =\sum \bar{b}_{u} \circ \bar{b}_{v} \circ b_{w} * f_{\beta} \\
& =\cdots=\sum \bar{b}_{x} \circ \bar{b}_{y} \circ b_{z} * f_{\gamma}=0 .
\end{aligned}
$$

So the lemma now follows immediately from Lemma 10.

We have now shown that the set $S_{r}$ forms a free set of generators for the $Z T$-module $\gamma_{r} F$ and hence completed the proof of Theorem $2^{\prime}$.

5. Consequences. We shall derive some consequences to the above theorems. To begin we state, without proof, the following:

LeMma 12. Let $H$ be a two-generator group, say $H=g p(a, b)$. Then $H$ is free if and only if the set $\left\{b_{j}=a^{-j} b a^{-j} \mid j \in Z\right\}$ freely generates a free group.

Now we prove

Theorem 3. Let $1 \rightarrow F \rightarrow G \rightarrow T \rightarrow 1$ be a short exact sequence where $F$ is parafree and $T=\langle t\rangle$ is infinite cyclic. If $F / F^{\prime}$ is a free $Z T$-module, then every two-generator subgroup of $G$ is free.

Proof. Let $H$ be any two-generator subgroup of $G$, say $H=g p(a, b)$. If $a \in F$, $b \in F$ then $H$ belongs to $F$ and hence is free. We divide the remainder of the proof into two cases.

Case 1. $a \in G \backslash F, b \in F$.

(i) $a=t^{n}, b \in F$.

We may assume that $b \in \gamma_{r} F$ but $b \notin \gamma_{r+1} F$ where $r \geqslant 1$. But by Theorem 2, $\gamma_{r} F / \gamma_{r+1} F$ is a free $Z T$-module. Hence modulo $\gamma_{r+1} F$, we have

$$
b * \sum_{\text {finite }} e_{i} t^{i}=0 \text { if and only if } e_{i}=0 \text { for all } e_{i}
$$

i.e. $\Pi\left(t^{-i} b t^{i}\right)^{e_{i}} \equiv 1$ if and only if $e_{i}=0$ for all $e_{i}$ i.e., modulo $\gamma_{r+1} F$, the set $\left\{b_{i}=t^{-i} b t^{i}, i \in Z\right\}$ is independent over $Z$. This implies that $g p\left(b_{i}=t^{-i} b t^{i} \mid i \in Z\right)$ is free on the $b_{i}$ 's. Hence by Lemma 12 , the group $H=g p(a, b)$ is free.

(ii) $a=t^{n} f, b \in F$ where $1 \neq f \in F$.

Observe that modulo $\gamma_{r+1} F$ we have $\left(t^{n} f\right)^{-1} b\left(t^{n} f\right) \equiv t^{-n} b t^{n}$ since $b \in \gamma_{r} F$ by assumption. So this subcase can be reduced to the previous subcase. 
Case 2. $a \in G \backslash F, b \in G \backslash F$.

We shall reduce this case to Case 1 . Let $a=t^{n_{1}} f_{1}, b=t^{n_{2}} f_{2}$ where $f_{1}, f_{2} \in F, n_{1}$, $n_{2} \in Z$. We can further assume $n_{1}>0, n_{2}>0$. Then

$$
H=g p(a, b)=g p\left(t^{n_{1}} f_{1}, t^{n_{2}} f_{2}\right) \text {. }
$$

If $n_{1}=n_{2}$, then

$$
H=g p\left(t^{n_{1}} f_{1}, t^{n_{2}} f_{2}\left(t^{n_{1}} f_{1}\right)^{-1}\right)=g p\left(t^{n_{1}} f_{1}, f\right)
$$

where $f \in F$ and we are in Case 1. If $n_{1}<n_{2}$, then choose $k$ such that $n_{2}=k n_{1}+$ $n_{3}$ with $0<n_{3}<n_{1}$. Then

$$
H=g p\left(t^{n_{1}} f_{1}, t^{n_{2}} f_{2}\left(t^{n_{1}} f_{1}\right)^{-k}\right)=g p\left(t^{n_{1}} f_{1}, t^{n_{3}} f\right)
$$

where $f \in F$. Now repeating this process we find

$$
H=g p\left(t^{n} g, h\right), \quad g, h \in F,
$$

and we are again in Case 1. This completes the proof of the theorem.

Similarly we have

TheOREM 4. Let $1 \rightarrow F \rightarrow G \rightarrow T \rightarrow 1$ be a short exact sequence where $F$ is parafree and $T=\langle t\rangle$ is infinite cyclic. If $F / F^{\prime}$ is a finitely generated free $Z T$-module freely generated by $\left\{c F^{\prime}, d F^{\prime}, \ldots, e F^{\prime}\right\}$, then the group $H=g p(t, c, d, \ldots, e)$ is free.

Proof. Notice that the set $\left\{t^{-i} c t^{i}, t^{-i} d t^{i}, \ldots, t^{-i} e t^{i} ; i \in Z\right\}$ freely generates a free group and hence the theorem follows.

We next show

TheOREM 5. Let $1 \rightarrow F \rightarrow G \rightarrow T \rightarrow 1$ be a short exact sequence where $F$ is parafree and $T=\langle t\rangle$ is infinite cyclic. If $F / F^{\prime}$ is a free $Z T$-module and $N$ is a finitely generated normal subgroup of $G$ contained in $F$, i.e., $N \triangleleft G, N \leqslant F$, then $N$ is trivial, i.e., $N=1$.

Proof. Suppose $N \neq 1$. Then there exists an $i \geqslant 1$ such that $N<\gamma_{i} F, N \Varangle$ $\gamma_{i+1} F$. Then $N \gamma_{i+1} F / \gamma_{i+1} F$ is a submodule of $\gamma_{i} F / \gamma_{i+1} F$ and $N \gamma_{i+1} F / \gamma_{i+1} F$ is a finitely generated abelian group since $N \gamma_{i+1} F / \gamma_{i+1} F \cong N / N \cap \gamma_{i+1} F$.

Now let $a \in N, a \notin \gamma_{i+1} F$. Then working modulo $\gamma_{i+1} F$, the conjugates of $a$ by the powers of $t$ are independent since $\gamma_{i} F / \gamma_{i+1} F$ is a free $Z T$-module. Hence $N \gamma_{i+1} F / \gamma_{i+1} F$ contains a free abelian group of infinite rank. This is a contradiction and hence $N=1$.

6. An example. We prove the following example (see G. Baumslag [7]).

THEOREM 6. Let $i$ and $j$ be nonzero integers and

$$
G=\left\langle a, b, c ; a=\left[c^{i}, a\right]\left[c^{j}, b\right]\right\rangle
$$

Then (i) $G$ is parafree.

(ii) $G$ is the third term of a short exact sequence $1 \rightarrow F \rightarrow G \rightarrow T \rightarrow 1$ where $F$ is free, $T$ is infinite cyclic and $F / F^{\prime}$ is a free $Z T$-module.

(iii) $G$ is not free. 
Proof. Let $F=g p_{G}(a, b)$. By the Reidemeister-Schreier rewriting procedure (see W. Magnus, A. Karrass and D. Solitar [21]),

$$
\begin{array}{r}
F=\left\langle\ldots, a_{-1}, a_{0}, a_{1}, \ldots, \ldots, b_{-1}, b_{0}, b_{1}, \ldots ;\right. \\
\left.\ldots, a_{n}=a_{n+i}^{-1} a_{n} b_{n+j}^{-1} b_{n}, \ldots\right\rangle
\end{array}
$$

where $a_{k}=c^{-k} a c^{k}, b_{k}=c^{-k} b c^{k}, k \in Z$. We may assume $j>0$ and it follows that $F$ is a free group freely generated by the elements $\ldots, a_{-1}, a_{0}, a_{1}, \ldots, b_{0}$, $b_{1}, \ldots, b_{j-1}$. Obviously $G / F$ is free cyclic on $c F^{\prime}$.

Again from the defining relations of $F$, it follows that $F / F^{\prime}$ is a free abelian group freely generated by $\ldots, b_{-1}, b_{0}, b_{1}, \ldots$ Hence $F / F^{\prime}$ is a free $Z T$-module where $T=\langle c\rangle$.

By Theorem 1, $G$ is parafree and, by $G$. Baumslag [5], $G$ is not free.

\section{REFERENCES}

1. B. Baumslag, Intersections of finitely generated subgroups in free products, J. London Math. Soc. 41 (1966), 673-679.

2. __ Generalised free products whose two-generator subgroups are free, J. London Math. Soc. 43 (1968), 601-606.

3. G. Baumslag, On generalised free products, Math. Z. 78 (1962), 423-438.

4. __ Groups with the same lower central sequence as a relatively free group. I. The groups, Trans.

Amer. Math. Soc. 129 (1967), 308-321.

5. __ Some groups which are just about free, Bull. Amer. Math. Soc. 73 (1967), 621-622.

6. ___ More groups which are just about free, Bull. Amer. Math. Soc. 74 (1968), 752-754.

7. $\ldots$ Groups with the same lower central sequence as a relatively free group. II. Properties, Trans.

Amer. Math. Soc. 142 (1969), 507-538.

8. __ Lecture notes on nilpotent groups, CBMS Regional Conf. Series in Math., no. 2, Amer. Math. Soc., Providence, R. I., 1971.

9.

Soc. 5 (1971), 87-94.

10. Bull. Austral. Math. Soc. 6 (1972), 313-314.

11.

Proc. Amer. Math. Soc. 35 (1972), 61-62.

12. Some problems on one-relator group, Proc. Second Internat. Conference on Theory of Groups, Lecture Notes in Math., vol. 372, Springer-Verlag, Berlin and New York, 1973, pp. 75-81.

13. G. Baumslag and U. Stammbach, $A$ non-free parafree group all of whose countable subgroups are free, Math. Z. 148 (1976), 63-65.

14. R. G. Burns, On the finitely generated subgroups of an amalgamated product of two groups, Trans. Amer. Math. Soc. 169 (1972), 293-306.

15. M. Hall, The theory of groups, Macmillan, New York, 1959.

16. P. Hall, The Edmonton notes on nilpotent groups, Queen Mary College Mathematics Notes, Mathematics Department, Queen Mary College, London, 1969.

17. N. Jacobson, Structure of rings, Amer. Math. Soc. Colloq. Publ., no. 37, Amer. Math. Soc., Providence, R. I., 1956.

18. A. Karrass and D. Solitar, On groups with one defining relation having an abelian normal subgroup, Proc. Amer. Math. Soc. 23 (1969), 5-10.

19. __ On finitely generated subgroups of a free product, Math. Z. 108 (1969), 285-287.

20. Math. Soc. 150 (1970), 227-255.

21. W. Magnus, A. Karrass and D. Solitar, Combinatorial group theory, Interscience, New York, 1966.

22. O. Schreier, Die Untergruppen der freien Gruppen, Abh. Math. Sem. Univ. Hamburg 5 (1928), 161-183.

23. P. C. Wong, On cyclic extensions of parafree groups, Ph. D. thesis, New York University, 1978.

Department of Mathematics, University of Malaya, Kuala Lumpur 22-11, Malaysia 